\title{
Simulation of the binary hard-sphere crystal/melt interface
}

\author{
Ruslan L. Davidchack and Brian B. Laird \\ Department of Chemistry, University of Kansas, Lawrence, Kansas 66045
}

(Received 5 June 1996)

\begin{abstract}
We report results of molecular-dynamics simulations on a planar binary hard-sphere disordered facecentered-cubic [100] crystal/melt interface. From the analysis of the single-particle density and diffusion profiles for the separate components, several phenomena are observed. The density profile for the smaller particle has an envelope that is decidedly nonmonotonic, unlike the same quantity for single-component systems. Also, the coarse-scaled density profiles show that the mole fractions of the two coexisting phases change from crystal-like to meltlike values over a length scale that is much shorter than that of the overall density, which exhibits a pronounced deficit in the interfacial region, indicating inefficient wetting of the [100] crystal surface by the fluid. [S1063-651X(96)50112-1]

PACS number(s): 68.45.-v, 61.90.+d, 64.70.Dv, 68.35.Fx
\end{abstract}

Despite its obvious importance in understanding the phenomena of near-equilibrium crystal growth and homogeneous nucleation, information about the microscopic nature of the interface between a crystal and its melt is sparse. This is primarily due to the near total lack of experimental data on such systems - the interface is sandwiched between two condensed phases with nearly identical densities, making study difficult [1]. Thus, in addition to the traditional roles of computer simulation in the interpretation of experimental results and in the evaluation and development of theoretical methods, simulations are also important in establishing the phenomenology of such interfacial systems [2].

Given that most crystals grown for technical applications must contain a specific concentration of some particular solute in order to possess the desired properties, it is somewhat surprising that, until now, interfaces of single-component systems have been the sole object of simulational studies (for a review of these studies, see Ref. [2]). In addition to issues of structure, dynamics, and thermodynamics that have been previously addressed in the context of single-component systems, multicomponent systems can exhibit interesting phenomena relating to differences (if any) in the density (concentration) and diffusion profiles of the separate species through the interfacial region. Such information would be important in obtaining a microscopic understanding of the phenomena of solute segregation $[1,3,4]$.

In this paper we present results for crystal/melt moleculardynamics [5] simulations of a multicomponent system, namely, a binary system composed of hard spheres with two different diameters. This system was chosen for this simulation for three reasons. First, since the structure and dynamics of simple dense systems is determined largely by the repulsive part of the interparticle potential, hard core systems are useful reference systems. Second, the coexistence concentrations for the binary hard-sphere systems for a variety of diameter ratios has been determined [6], so the boundary conditions for the simulations are already known. Third, because of the availability of accurate analytical approximations for fluid structure and thermodynamics, calculation hard-sphere systems are important benchmarks in testing densityfunctional theories of the interface [7-9].
Simulations of crystal/melt interfaces generally focus on two quantities that characterize the structure and dynamics within the interfacial region. The first is the single-particle density, which is inhomogeneous and periodic in the crystal and uniform in the bulk fluid. One normally visualizes this quantity by averaging over the directions perpendicular to the interface normal (taken to be $z$ ) to generate the singleparticle density profile

$$
\rho_{i}(z)=\left\langle\rho_{i}(\vec{r})\right\rangle_{x, y},
$$

where $i$ indexes the particle type in a multicomponent system. Mass transport in the interface is best quantified through the diffusion-constant profiles $D_{i}(z)$ (defined later), which are especially important in the understanding of nearequilibrium crystal growth, since they determine the time scale of molecular rearrangement at the growing interfacial front. A third quantity, the interfacial free energy, is important in characterizing the thermodynamics of the interface, but is extremely difficult to calculate, either in a simulation or experimentally $[2,10]$, and will not be considered in this current work.

We consider a two-component system made of hardsphere particles. The two types of particles (labeled 1 and 2) are distinguished by their differing diameters, $\sigma_{1}$ and $\sigma_{2}$ (their masses are assumed identical). We assume, without loss of generality, that $\sigma_{2}>\sigma_{1}$, and we measure all length scales in terms of the diameter of the larger particle, $\sigma_{2}$. Thus, the thermodynamic state of the bulk system is completely characterized by the total number density $\rho$, the mole fraction $x_{2}$ of the larger particle, and the diameter ratio $\alpha=\sigma_{1} / \sigma_{2}$. Depending upon the value of $\alpha$, the binary hardsphere system can exhibit a variety of equilibrium crystal phases $[11,12]$. Below about $\alpha=0.85$ ordered solids of either the $\mathrm{NaCl}$ or $\mathrm{CsCl}$ type are found to be the stable phases at freezing. For $\alpha>0.85$, however, the stable crystal phase is a substitutionally disordered face-centered-cubic (fcc) crystal. It is this structure that is the focus of the present work.

Kranendonk and Frenkel [6] have determined the fluiddisordered-fcc phase diagram for hard-sphere mixtures for $\alpha=0.9$ and 0.95 . To maximize the deviation from single- 
component behavior, we have chosen for this study a point on the $\alpha=0.9$ phase diagram where the mole fraction differences between the crystal and melt phase are maximum. This point occurs at a pressure of $P=14.7 k T / \sigma_{2}^{3}$ and the mole fractions of the larger particles are $x_{2}^{(c)}=0.714$ and $x_{2}^{(f)}=0.550$, where the superscripts $c$ and $f$ denote crystal and fluid phase values, respectively. The total number densities in each two phases at this pressure were $\rho^{(c)}=1.145$ and $\rho^{(f)}=1.097 \sigma_{2}^{-3}$. The construction of an interface must be carried out carefully to insure that the system will remain stable and at coexistence. Because of the importance of this procedure, we will outline our method in some detail.

We first prepared separate crystal and fluid bulk systems at the desired densities and mole fractions. The fcc [100] crystal/melt interface was set up parallel to the $x-y$ plane. The system consisted of a total of 12000 particles. The crystal block consisted of 30 square layers, each containing 200 spheres. The fluid block, containing 6000 spheres, was given the same $x y$ cross-sectional area as the crystal block, but was slightly expanded in the $z$ direction, to yield the proper number densities. In the fluid, the two sphere types were distributed randomly throughout the relevant volume, while in the crystal randomization it was done layer by layer, thereby removing layer-to-layer concentration fluctuations due to finite system size - since each crystal layer in our simulations contained more particles than were used to establish the bulk crystal-fluid coexistence [6], this constraint is not expected to affect the results in any significant way.

Unlike in simulations of continuous potentials where the atoms in the initial fluid configuration can be placed randomly within the simulation cell, hard spheres are best set up in an ordered manner to ensure a nonoverlapping initial configuration. The system is evolved until the initial order disappears. To speed up the fluid equilibration, we first created a fluid phase at a density about $20 \%$ below coexistence, and then gradually increased the sphere diameters until the desired density was reached. The degree of order was monitored by the means of the Verlet translational order parameter $\lambda[13]$.

To ensure that the particles in the fluid configuration do not have overlap with those at the crystal surface when the interface is constructed, the fluid density was increased to slightly above the coexistence value and placed next to the crystal block with a small gap in between so that there was no overlap. Then the fluid particles were assigned velocities according to a Maxwell distribution and were allowed to move with the particles in the crystal held fixed. The gap between the blocks was such that, after filling this gap, the average total density of the fluid decreased to $\rho^{(f)}$.

Two different tests were used to optimize the gap width. First, the pressure in the fluid was required to be equal to the coexistence pressure, while the crystal particles were held fixed. Second, after the combined system was allowed to reach equilibrium, the lattice plane spacing of the bulk crystal was checked to ensure that no expansion or contraction of the bulk crystal had occurred which would move the system away from coexistence.

In the final stage of the construction all velocities were reassigned and the system was allowed to evolve until equilibrium was reached. To improve the statistics of our results

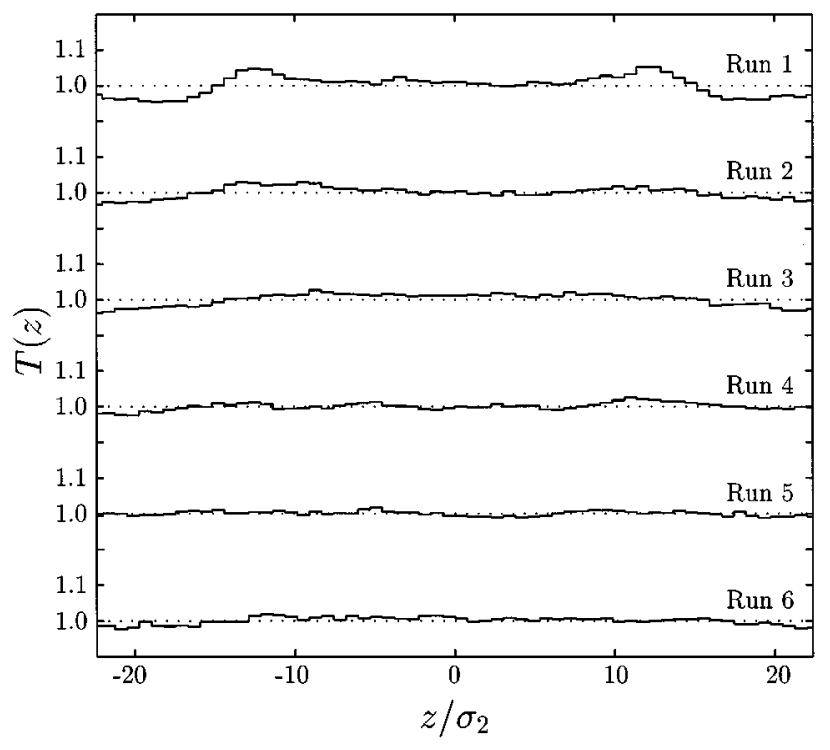

FIG. 1. Evolution of the temperature profile during the process of equilibration. In each run the systems are allowed to evolve for 500000 collisions.

we created ten different crystal/melt interfaces under identical conditions, but using different starting configurations. The systems were then allowed to evolve under identical circumstances and all results were averaged over the ten systems.

To characterize the interface and monitor the approach to equilibrium, all averaged quantities (densities, diffusion constants, pressure, temperature) were calculated as functions of the coordinate $(z)$ normal to the interfacial plane. These $x$ $y$ averaged parameter profiles were generated by partitioning the $z$ axis into discrete bins. Two different bin sizes were utilized. For the coarse scale the width of the bins was set to the bulk crystal-plane spacing. For the fine scale each coarse scale bin was divided into 25 equal parts. These bin widths $\Delta z$ were equal to $0.7588 \sigma_{2}$ and $0.0304 \sigma_{2}$ for the coarse and fine scales, respectively.

Temperature evolution during the equilibration process is shown in Fig. 1 (the solid occupies the center of the $z$ range). The coarse-scaled temperature profile, monitored during six consecutive equilibration runs of 500000 collisions each, exhibits an initial heating at the interface that tends to dissipate mostly into the crystal, resulting in about 5\% temperature difference between the fluid and the crystal parts of the system, which was diminished rather slowly. In order to eliminate this temperature gradient, the velocities were randomized once more after the third equilibration run. The next three runs show the temperature to be uniform within random fluctuations. The transverse pressure profiles showed similar behavior, ensuring mechanical equilibrium. Following equilibration, the systems were evolved for 2000000 collisions, and all the final diagnostics and averages are calculated during this run. Thus produced, the interface was stationary and showed remarkable stability over long runs and the two interfaces were identical within statistical error. For the density and diffusion profiles that follow, the profiles were folded over so that the interfacial peaks coincided, and then final averages were taken over two interfaces.

As mentioned earlier, the interfacial structure can be char- 


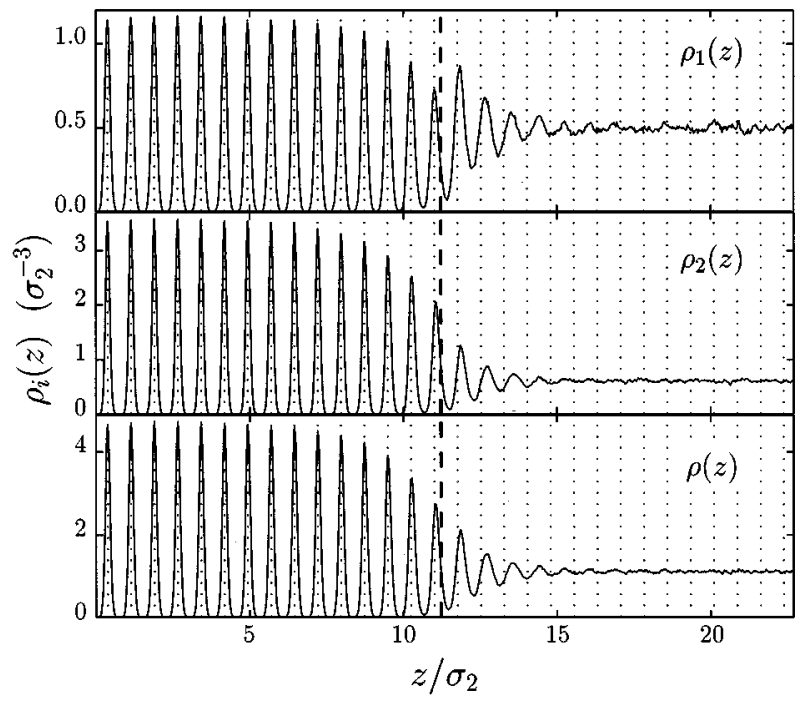

FIG. 2. Density profiles for the binary hard-sphere fcc [100] crystal/melt interface plotted on the fine scale with the bin width equal to 0.04 times the crystal layer spacing. The dashed vertical line is the Gibbs dividing surface as defined in the text. The dotted grid is commensurate with the lattice planes in the bulk crystal and is included to better visualize the expansion of the lattice constant in the interfacial region.

acterized by the single-particle density profiles. These profiles reveal interesting features on both fine and coarse scales. The fine-scale profiles for the two separate components $\rho_{1}(z), \quad \rho_{2}(z)$ and for the total density $\rho(z)=\rho_{1}(z)+\rho_{2}(z)$ are shown in Fig. 2. [In this plot and those that follow we indicate by a dashed vertical line the Gibbs dividing surface defined such that the excess number of solvent (type 2) particles is zero. This gives a useful guide in examining the spatial relationship between various features in the plots.] In the crystal region there are large oscillations corresponding to the crystal layers. In the interfacial region, the density oscillations in $\rho_{2}(z)$ and $\rho(z)$ dampen gradually over nine or ten atomic layers, while $\rho_{1}(z)$ exhibits a peculiar nonmonotonic peak-height envelope, a phenomenon that has not been seen in any of the singlecomponent system studies. (Note that this feature was also present in a separate simulation of 3000 particles with a smaller cross-sectional area, which indicates that it is not an artifact of the specific system size.) This behavior can be explained by examining the density profiles on a coarse scale. These are shown in Fig. 3. This figure shows that the concentrations of the two species change from crystal-like to fluidlike values over a relatively short length scale-much shorter than indicated by the fine-scaled densities. Since the density of the smaller spheres in the fluid $\left[\rho_{1}^{(f)}\right.$ $\left.=\left(1-x_{2}^{(f)}\right) \rho^{(f)}=0.494 \sigma_{2}^{-3}\right]$ is larger than the corresponding number density in the crystal region $\left[\rho_{1}^{(c)}\right.$ $\left.=\left(1-x_{2}^{(c)}\right) \rho^{(c)}=0.323 \sigma_{2}^{-3}\right]$, the ordering of the fluid in the presence of the interface occurs in a region with a higher bulk density of type 1 (smaller) particles than that in the bulk crystal, resulting in the profile peaks due to this ordering being higher than the profile peaks in crystal.

Another feature seen in the fine-scaled interfacial density profiles is that the crystal layers tend to relax outward into

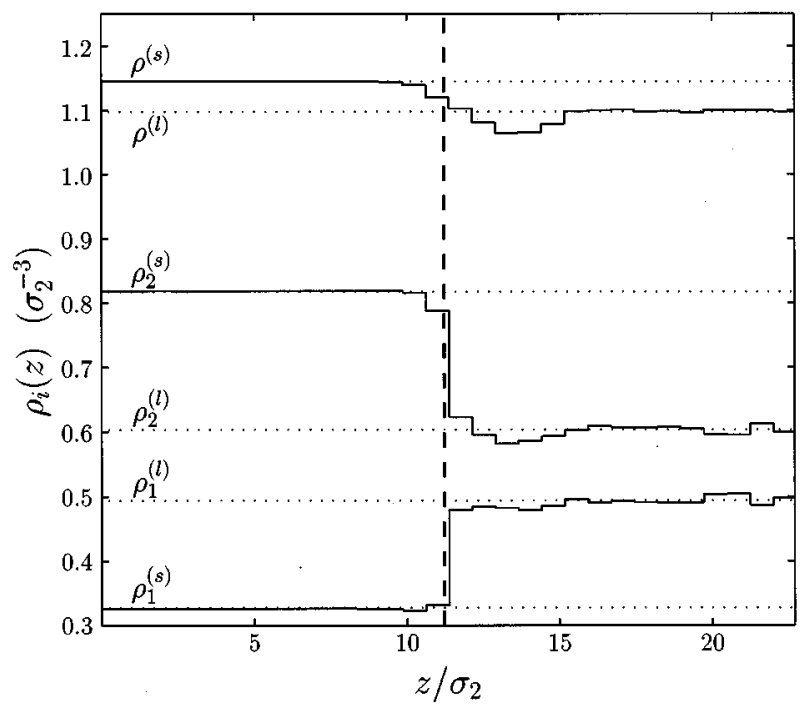

FIG. 3. Density profiles on the coarse scale. The bin width is equal to the crystal layer spacing. The density oscillations in the crystal region are averaged over and the density deficit in $\rho(z)$ can be seen at the interface. The dashed vertical line is the Gibbs dividing surface as defined in the text.

the fluid region, so that the spacing of the peaks in the density oscillations increases. This phenomenon has also been observed in recent Monte Carlo [14] and moleculardynamics [15] simulations of the single-component hardsphere interface, where it was interpreted as a preference of the fluid to order at a planar interface in a way that is more consistent with a [111] face. A more detailed investigation of this feature is underway.

As the density oscillations are averaged over on the coarse scale (Fig. 3), a deficit in the total density can be seen in the interfacial region indicating inefficient wetting of the [100] surface by the fluid. (Note that this feature has a spatial extent several times larger than the bin spacing and therefore cannot be an artifact due to the fact that the bin spacing becomes incommensurate with the lattice spacing in the interfacial region.) A similar, but not as pronounced, deficit can be seen in plots of the coarse-scaled density in simulations of the single-component Lennard-Jones fcc[100]/liquid interface [16]. This inefficient wetting of the [100] surface is most probably due to the same effect that leads to the above mentioned increase in the planar spacing as the interface is approached. This deficit should not be confused with that found in early experiments and computer models using a Bernal random close-packing model for the singlecomponent hard-sphere fcc[111]/fluid interface $[17,18]$. These results were later shown to be an artifact of the lack of any relaxation in the Bernal model [10]. In our work we see that this feature of the interface is stable for relatively long runs (up to 15000000 collisions) for both binary and singlecomponent [19] systems.

We also computed the diffusion constant profiles for both types of spheres during the averaging runs (see Fig. 4). The diffusion constant profiles are defined as

$$
D_{i}(z)=\frac{1}{6 N_{i}(z)} \frac{d}{d t} \sum_{j=1}^{N_{i}(z)}\left\langle\left[\mathbf{r}_{j}(t)-\mathbf{r}_{j}\left(t_{0}\right)\right]^{2}\right\rangle
$$




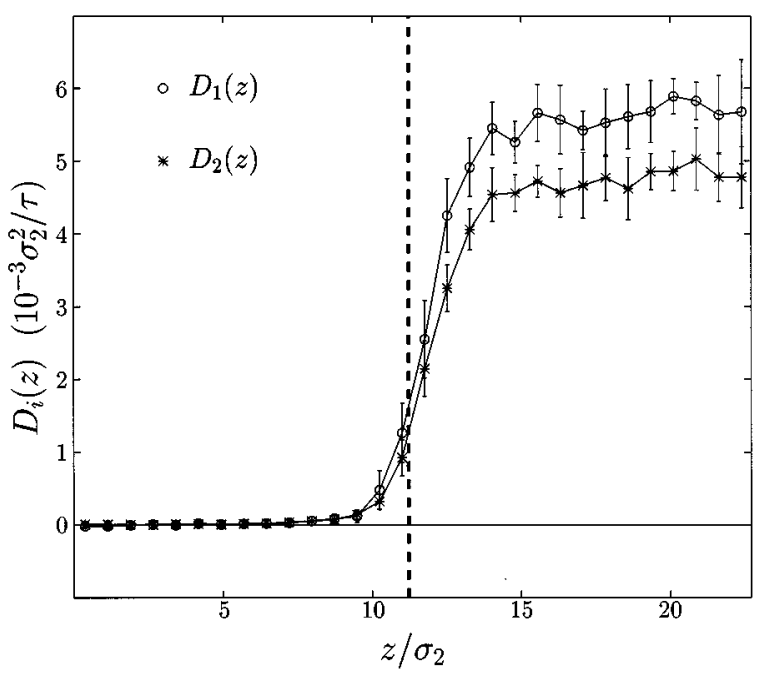

FIG. 4. Diffusion coefficient profiles. Error bars represent the standard deviations calculated from the diffusion coefficient values obtained for ten different systems. The dashed vertical line is the Gibbs dividing surface as defined in the text.

where $N_{i}(z)$ is the number of spheres of type $i$ between $z-\Delta z / 2$ and $z+\Delta z / 2$ at time $t=t_{0}$, and the brackets represent the average over time origins $t_{0}$. The sphere displacement was monitored over time $t_{\max }-t_{0}=60 \tau$, where $\tau$ is the average collision time. During this time the average fluid particle will be displaced about 1 particle diameter, so this bin assignment should remain valid for the entire process. The averaging was done over about 100 time origins. The 10-90 width of an interfacial profile is defined to be the length scale over which the relative deviation of the property being measured from its value in the crystal phase changes from $10 \%$ to $90 \%$. For the diffusion constants this width is the same for both types of particles and can be estimated to be about $3.2 \sigma_{2}$.

In summary we have performed simulations of a multicomponent crystal/melt interface. These simulations on the binary hard-sphere system show several novel features. First, the fine-scaled single-particle density profile of the smaller particles possesses a decidedly nonmonotonically decreasing envelope of density oscillations as the interface is traversed from crystal to melt. This was shown to be due to the spatially narrow change in the concentration profiles from crystal to melt values in combination with the usual ordering of the melt near the interface. Also, a pronounced deficit in the total coarse-scaled density is observed at the interface indicating poor wetting of the [100] fcc surface by the fluid mixture. These qualitative features, as well as those for other crystal faces (calculations of which are underway), will be important in the development of a density-functional theory of such interfacial systems as they indicate the minimum complexity that parametrizations of the single-particle density must possess to be successful.

The authors gratefully acknowledge support from the National Science Foundation under Grant No. CHE-950281.
[1] D.P. Woodruff, The Solid-Liquid Interface (Cambridge University Press, London, 1973).

[2] B.B. Laird and A.D.J. Haymet, Chem. Rev. 92, 1819 (1992).

[3] D.T.J. Hurle, Crystal Pulling from the Melt (Springer-Verlag, New York, 1993).

[4] J.R. Carruthers and A.F. Witt, Crystal Growth and Characterization (Elsevier, New York, 1975).

[5] M.A. Allen and D.J. Tildesley, Computer Simulation of Liquids (Oxford Science Press, Oxford, 1987).

[6] W.G.T. Kranendonk and D. Frenkel, J. Phys. Condens. Matter 1, 7735 (1989).

[7] D.W. Marr and A.P. Gast, Phys. Rev. E 47, 1212 (1993).

[8] W.A. Curtin, Phys. Rev. B 39, 6775 (1989).

[9] W.E. McMullen and D.W. Oxtoby, J. Chem. Phys. 88, 1967 (1988).

[10] J.Q. Broughton and G.H. Gilmer, J. Chem. Phys. 84, 5759 (1986)
[11] W. Hume-Rothery, R.E. Smallman, and C.W. Haworth, The Structure of Metals and Alloys (The Metals and Metallurgy Trust, London, 1969).

[12] J.L. Barrat, M. Baus, and J.P. Hansen, Phys. Rev. Lett. 56, 1063 (1986).

[13] L. Verlet, Phys. Rev. 159, 98 (1967).

[14] A. Kyrlidis and R.A. Brown, Phys. Rev. E 51, 5832 (1995).

[15] A. Mori, R. Manabe, and K. Nishioka, Phys. Rev. E 51, R3831 (1995).

[16] J.Q. Broughton, A. Bonissent, and F.F. Abraham, J. Chem. Phys. 44, 4029 (1981).

[17] J.D. Bernal, Proc. R. Soc. London, Ser. A 280, 299 (1964).

[18] A. Bonissent, Interfacial Aspects of Phase Transitions (Reidel, Boston, 1982).

[19] R.L. Davidchack and B.B. Laird (unpublished). 